\title{
Non-cultural detection and molecular genotyping of Neisseria gonorhoeae from a piece of clothing
}

\begin{abstract}
Correspondence
Catherine A. Ison

catherine.ison@hpa.org.uk
\end{abstract}

Received 19 September 2006

Accepted 24 November 2006

\author{
Iona M. C. Martin, ${ }^{1}$ Ellie Foreman, ${ }^{2}$ Vicky Hall, ${ }^{2}$ Anne Nesbitt, ${ }^{3}$ \\ Greta Forster ${ }^{3,4}$ and Catherine A. Ison ${ }^{1}$ \\ ${ }^{1}$ Sexually Transmitted Bacteria Reference Laboratory, Health Protection Agency Centre for \\ Infections, 61 Colindale Avenue, London NW9 5EQ, UK \\ ${ }^{2}$ Forensic Science Service, 109 Lambeth Road, London SE1 7LP, UK \\ ${ }^{3}$ The Haven, Brady Street, Whitechapel, London E1, UK \\ ${ }^{4}$ Genitourinary Medicine, Barts and the London NHS Trust, London, UK
}

\begin{abstract}
Isolation of Neisseria gonorrhoeae is currently the gold standard for the definitive diagnosis of gonorrhoea and for use in medico-legal cases in the UK. Molecular detection methods are used increasingly but are untested as evidence of infection in a court of law. An isolate of $N$. gonorrhoeae was obtained from a child and an article of clothing from an adult male who was suspected of sexual abuse of the child. Biochemical and immunological tests were used to confirm the isolate as $N$. gonorrhoeae. Amplification by PCR using two targets, $c p p B$ and omplll, was used both as further confirmation of the isolate and to detect the presence of gonococcal-specific DNA from the clothing. The relationship of the gonococcal DNA from the child and the adult was investigated using genotyping (N. gonorrhoeae multi-antigen sequence typing; NG-MAST), including a nested PCR for the por gene. Both samples were indistinguishable by NG-MAST and shared the same sequence type, 403. This is the first report of molecular detection and genotyping of $N$. gonorrhoeae on an article of clothing, which resulted in conviction of the man for sexual assault.
\end{abstract}

\section{INTRODUCTION}

Neisseria gonorrhoeae causes the sexually transmitted infection gonorrhoea and is almost exclusively transmitted by sexual intercourse in adults. Currently, isolation and identification of the causative organism remains the gold standard for a definitive diagnosis. However, nucleic-acidbased techniques are being used more frequently as sensitivity and specificity of the newer tests are improved (Palmer et al., 2003), although none have been licensed for use with specimens from non-genital sites, including the rectum and the pharynx. There is also little validation of molecular methods for diagnosis of gonorrhoea in children and they should be used with caution as the prevalence of infection is likely to be low (Hammerschlag, 2001).

In medico-legal cases, such as sexual abuse of a child or rape, it is necessary not only to confirm the presence of the infecting organism but also to attempt to prove linkage between the isolates from each individual involved. Typing of gonococcal isolates has been used for this purpose because it can support or dispute the likelihood of transmission between individuals, and newer molecular methods offer

Abbreviations: NG-MAST, Neisseria gonorrhoeae multi-antigen sequence typing; ST, sequence type; STBRL, Sexually Transmitted Bacteria Reference Laboratory. greater discrimination compared to phenotypic methods (Ison, 1998). A genotypic method needs to be based on a target that accumulates genetic variation quickly, so that individuals that are sexual contacts or part of a short transmission chain should demonstrate an indistinguishable type, while individuals that are unlinked should have different types. N. gonorrhoeae multi-antigen sequence typing (NG-MAST) is a sequence-based method examining the diversity in two outer-membrane proteins, Por and $\mathrm{TbpB}$ (transferrin binding protein $\mathrm{B}$ ), allowing the precise characterization and comparison of gonococcal samples, with all analysis performed via a website (http://www.ngmast.net). The method has been shown to identify epidemiologically linked individuals and is able to distinguish between individuals that are not linked (Martin et al., 2004). Ideally, variation in hyper-variable genes, resulting from genetic exchange and recombination during mixed infection, should produce a changing population of molecular types over time. Highly diverse populations have been identified (Choudhury et al., 2006) but persistence of molecular types within a population has also been shown (Ward et al., 2000) and may reflect a lower rate of partner change and hence chance of mixed infection.

The Sexually Transmitted Bacteria Reference Laboratory (STBRL) at the Health Protection Agency Centre for Infections uses molecular methods for confirmation of 
identity and typing when isolates are received from potentially linked sources, to support or dispute the likelihood of transmission of gonorrhoea. This short report describes the first use of molecular methods to confirm the presence of $N$. gonorrhoeae from adult clothing and provide evidence of possible linkage to an isolate from a child.

\section{METHODS}

Specimens received. In February 2005, the STBRL received a strain of N. gonorrhoeae isolated from a low vaginal swab from a child for confirmation for medico-legal reasons. In April 2005, the Forensic Science Laboratory received a pair of underpants from an adult male for detection of N. gonorrhoeae. Six areas of visible staining from the inside front of the underpants and two clean areas from the inside back of the waistband were excised. The samples of material were soaked separately for $30 \mathrm{~min}$ in $500 \mu \mathrm{l}$ molecular biology grade water then vortexed for $1 \mathrm{~min}$. All liquid was then transferred into fresh Eppendorf tubes. The extracts were split into two groups of four (three samples from a stained area and one from a clean area in each) and one set was delivered with a chain of evidence form to the STBRL.

Culture and identification. The isolate from the child was retrieved on GC agar base (Becton Dickinson) supplemented with $1 \%$ Vitox (Oxoid) and incubated overnight at $36^{\circ} \mathrm{C}$ with $5 \%$ carbon dioxide. Identification was performed using the Gram stain and oxidase test, followed by biochemical characterization using an APINH test (bioMérieux) and immunological confirmation using a Phadebact test (Boule) following the manufacturer's recommended method.

DNA extraction. A turbid suspension of the gonococcal culture was made in PBS. The bacteria were pelleted by centrifugation at $2000 \mathrm{~g}$ for $5 \mathrm{~min}$, washed once and resuspended in PBS, and boiled for $5 \mathrm{~min}$ to lyse the cells. The lysate was centrifuged for $5 \mathrm{~min}$ at $2000 \mathrm{~g}$ and the supernatant was stored at $-20^{\circ} \mathrm{C}$ until required.

PCR detection of gonococcal-specific DNA. The presence of $N$. gonorrhoeae specific DNA was determined using two in-house PCR methods targeting the ompIII and $\angle P p B$ genes using the DNA extract from the culture and the extracts from the underpants (Ho et al., 1992; Liebling et al., 1994). Negative and positive controls were included in each PCR performed.

Molecular typing by NG-MAST. Molecular typing using the sequence-based method NG-MAST was performed on the DNA extract from the culture and samples from the underpants (Martin et al., 2004). Briefly, internal regions of the por and $t b p B$ genes were amplified by PCR and both strands of DNA were sequenced using a Beckman 310. Sequences were aligned, edited and trimmed to a fixed length from conserved positions, as described previously (Martin et al., 2004). Alleles were assigned to each por and tbpB sequence and the corresponding sequence type (ST) was assigned from the combination of the alleles at the two loci using the NGMAST website (http://www.ng-mast.net).

Nested por PCR for NG-MAST. Primers internal to the original por primers were designed to amplify both classes of alleles (por IA and IB), using conserved sequences of the porin protein, amplifying a fragment of $652 \mathrm{bp}$. The primers used were PorInt-forward $5^{\prime}{ }^{381}$ TTT GGC AGT TGG AAC AA ${ }^{397}-3^{\prime}$ and PorInt-reverse $5^{\prime}-{ }^{1015}$ TTG AAG CCG TGG GCG TAA ${ }^{1071}-3^{\prime}$ (numbering is based on the por gene of strain MS11; GenBank accession no. M21289) (Carbonetti et al., 1988). PCR amplification of the por gene fragment was carried out in reaction volumes of $50 \mu \mathrm{l}$ with a PTC-200 DNA engine (MJ Research). Each PCR reaction contained $50 \mathrm{pmol}$ of each primer, $1 \times$ buffer (Invitrogen), $2.5 \mathrm{U}$ Taq polymerase (Invitrogen), $2 \mu \mathrm{l}$ round I por PCR product, $0.2 \mathrm{mM}$ each dNTP (Invitrogen) and water to $50 \mu \mathrm{l}$. The PCR cycle involved an initial denaturation at $95^{\circ} \mathrm{C}$ for $4 \mathrm{~min}$, followed by 25 cycles of $95^{\circ} \mathrm{C}$ for $30 \mathrm{~s}, 58^{\circ} \mathrm{C}$ for $30 \mathrm{~s}$ and $72^{\circ} \mathrm{C}$ for $1 \mathrm{~min}$, followed by a final extension of $72^{\circ} \mathrm{C}$ for $10 \mathrm{~min}$ and cooling to $4{ }^{\circ} \mathrm{C}$. Sequencing from the nested por PCR product proceeded as previously described but using the internal PCR primers for the sequencing reaction (Martin et al., 2004). Typing by NG-MAST using the nested primers had previously been shown to result in the same ST as the original nonnested primers on a previously described panel of 20 gonococcal isolates (data not shown).

\section{RESULTS AND DISCUSSION}

The isolate from the child was confirmed as being $N$. gonorrhoeae by the APINH (result 1001) and Phadebact (serovar 1B) tests and gonococcal-specific DNA was detected by amplification of the ompIII and $\operatorname{cppB}$ genes. Genotyping by NG-MAST resulted in ST 403 (por-295, $t b p B-16)$. The three samples of one set received from the forensic laboratory taken from the stained area of the underpants were positive for the presence of gonococcalspecific DNA using the PCR targeting both genes; the sample from the clean area was negative. The $t b p B$ gene in the three positive samples amplified sufficiently for sequencing, but the por gene did not produce sufficient PCR product and hence was amplified using the nested por PCR for sequencing. All three samples resulted in ST 403 (por-295, tbpB-16).

The optimum number and type of tests required by a court of law to confirm the identity of an isolate as $N$. gonorrhoeae in the UK have not been defined. Our approach has been to use a combination of biochemical, immunological and molecular tests for identification. This will guard against misidentification of an isolate due to an anomalous result with a single test, such as $N$. gonorrhoeae lacking the prolineiminopeptidase gene (Alexander \& Ison, 2005). The choice of molecular confirmation needs careful consideration to avoid false positive and negative results. In this study, we have used ompIII and $c p p B$ genes, but the use of the $c p p B$ gene is open to criticism as this assay is known to have problems, particularly in false negative results due to strains lacking this gene (Lum et al., 2005; Whiley et al., 2006). There are alternative in-house assays described and it would be our approach to use at least two of these in combination for medico-legal cases (Smith et al., 2005).

This is the first report using molecular methods for detection and typing of gonococcal DNA from a piece of clothing for medico-legal purposes, which we have shown can be achieved if the DNA load is sufficient for amplification. In this case, the adult male who was accused of sexual abuse of the child had refused to provide an invasive urethral sample when requested by the investigating police. However, he was required to remove his underpants, which were supplied to the Forensic Science Laboratory. The underpants were heavily stained from an apparent discharge, and sampling from these stained areas resulted in the 
elution of DNA that was subsequently confirmed as containing gonococcal-specific DNA. This result was confirmed by amplification of two genes (ompIII and $c p p B$ ) for detection and a further two (por and $t b p B$ ) for typing purposes and suggests that the discharge on the underpants is highly likely to have been from a genital infection of gonorrhoea. Amplification of different genes decreases the possibility of a non-specific result which may occur because of the close genetic relatedness of the species belonging to the Neisseria genus (Palmer et al., 2003). This is supported by the sequencing, which gives additional evidence of identity and supplies typing information. It may be necessary to add sequence data when using molecular detection or typing as evidence in a court of law as these methods have not been tested as forms of evidence, although it may be necessary to include a number of housekeeping genes to establish identity beyond reasonable doubt (Hanage et al., 2005).

Genotyping of the samples taken from the underpants and the isolate obtained from the child were indistinguishable by NG-MAST and shared the same ST, suggesting that they could have been acquired from a common source. If the genotyping had shown different types it would have been possible to dispute the possibility of sexual transmission between the child and the adult, but these results support the possibility of sexual activity although they cannot confirm direct contact. The NG-MAST database contains over 4000 strains and this ST had previously only been detected in an additional two strains, which were isolated in London during 2004. This information should be interpreted with caution as the database is not truly representative as it consists of results from isolates largely selected for specific studies and may not include background information of the gonococcal population found at a particular geographical location. Hence it may be unknown when two isolates share the same molecular type whether they are part of a short transmission chain, where the likelihood of linkage will be high, or a persistent cluster, where it will be less.

In children infected with gonorrhoea, sexual abuse should be assumed unless there is evidence to suggest otherwise and in the majority of cases this has been proven to have occurred (Neinstein et al., 1984). The vagina of prepubescent girls is much shorter than that of adults $(4-5 \mathrm{~cm}$ as opposed to $11-12 \mathrm{~cm}$ ) and is lined with columnar epithelia cells that gonococci attach to which is necessary to establish infection, causing vaginitis; adults have stratified squamous cells to which gonococci cannot attach. The vagina in children is also alkaline, compared to acidic in adults, and all of these factors allow $N$. gonorrhoeae to establish infection within the vagina in children but not in adults, where the primary site is the cervix (Jenny, 1992). It is therefore possible for transmission of gonorrhoea to occur in children by sexual intercourse, sexual abuse not involving sexual penetration and sex play.

When presented with this evidence, the perpetrator pleaded guilty to sexual assault before the case was brought to trial and was sentenced to 2 years' imprisonment based on no previous history of abuse. Molecular methods are now available to provide evidence of a sexually transmitted infection, but have yet to be fully tested in a court of law. They may be particularly useful when there has been a delay in reporting, which often occurs with children suffering abuse, and for use with specimens that have been stored and no longer contain viable organisms. However, nucleic acid amplification tests have not been licensed or validated for use with specimens from children and need to be used with caution in a population that is likely to have a low prevalence and result in false positive results. Extensive supplementary testing will be necessary for confirmation before the results will be sufficiently robust to provide evidence in a court of law.

\section{ACKNOWLEDGEMENTS}

We would like to thank Detective Constable Kevin O'Loughlin, the staff of The Haven, Whitechapel, the Microbiology Department of Barts and The London Hospital and of the Sexually Transmitted Bacteria Reference Laboratory for their contribution to this work.

\section{REFERENCES}

Alexander, S. \& Ison, C. (2005). Evaluation of commercial kits for the identification of Neisseria gonorrhoeae. J Med Microbiol 54, 827-831.

Carbonetti, N. H., Simnad, V. I., Seifert, H. S., So, M. \& Sparling, P. F. (1988). Genetics of protein I of Neisseria gonorrhoeae: construction of hybrid porins. Proc Natl Acad Sci U S A 85, 6841-6845.

Choudhury, B., Risley, C. L., Ghani, A. C., Bishop, C. J., Ward, H., Fenton, K. A., Ison, C. A. \& Spratt, B. G. (2006). Identification of individuals with gonorrhoea within sexual networks: a population based study. Lancet 368, 139-146.

Hammerschlag, M. R. (2001). Use of nucleic acid amplification tests in investigating child sexual abuse. Sex Transm Infect 77, 153-154.

Hanage, W. P., Fraser, C. \& Spratt, B. G. (2005). Fuzzy species among recombinogenic bacteria. BMC Biol 3, 6-13.

Ho, B. S. W., Feng, W. G., Wong, B. K. C. \& Egglestone, S. I. (1992). Polymerase chain reaction for the detection of Neisseria gonorrhoeae in clinical samples. J Clin Pathol 45, 439-442.

Ison, C. A. (1998). Genotyping of N. gonorrhoeae. Curr Opin Infect Dis 11, 43-46.

Jenny, C. (1992). Sexually transmitted diseases and child abuse. Pediatr Ann 21, 497-503.

Liebling, M. R., Arkfeld, D. G., Michelini, G. A., Nishio, M. K., Eng, B. J., Jin, T. \& Louie, J. S. (1994). Identification of Neisseria gonorrhoeae in synovial fluid using the polymerase chain reaction. Arthritis Rheum 37, 702-709.

Lum, G., Freeman, K., Nguyen, N. L., Limnios, E. A., Tabrizi, S. N., Carter, I., Chambers, I. W., Whiley, D. M., Sloots, T. P. \& other authors (2005). A cluster of culture positive gonococcal infections but with false negative $c p p B$ gene based PCR. Sex Transm Infect 81, 400-402.

Martin, I. M. C., Ison, C. A., Aanensen, D. M., Fenton, K. A. \& Spratt, B. G. (2004). Rapid sequence-based identification of gonococcal transmission clusters in a large metropolitan area. J Infect Dis 189, 1497-1505. 
Neinstein, L. S., Goldenring, J. \& Carpenter, S. (1984). Nonsexual transmission of sexually transmitted diseases: an infrequent occurrence. Pediatrics 74, 67-76.

Palmer, H. M., Mallinson, H., Wood, R. L. \& Herring, A. J. (2003). Evaluation of the specificities of five DNA amplification methods for ?the detection of Neisseria gonorrhoeae. J Clin Microbiol 41, ?835-837.

Smith, D. W., Tapsall, J. W. \& Lum, G. (2005). Guidelines for the use and interpretation of nucleic acid detection tests for Neisseria gonorrhoeae in Australia: a position paper on behalf of the Public Health Laboratory Network. Commun Dis Intell 29, 358-365.

Ward, H., Ison, C. A., Day, S. E., Martin, I. M., Ghani, A. C., Garnett, G. P., Bell, G., Kinghorn, G. \& Weber, J. N. A. (2000). A prospective social and molecular investigation of gonococcal transmission. Lancet 356, 1812-1817.

Whiley, D. M., Tapsall, J. W. \& Sloots, T. P. (2006). Nucleic acid amplification testing for Neisseria gonorrhoeae: an ongoing challenge. J Mol Diagn 8, 3-15. 